This item was submitted to Loughborough's Research Repository by the author.

Items in Figshare are protected by copyright, with all rights reserved, unless otherwise indicated.

\title{
Influence of the prefactor to defect motion in alpha-Iron during long time scale simulations
}

PLEASE CITE THE PUBLISHED VERSION

http://dx.doi.org/10.1088/0953-8984/26/39/395007

PUBLISHER

(C) IOP Publishing Ltd.

\section{VERSION}

AM (Accepted Manuscript)

\section{PUBLISHER STATEMENT}

This work is made available according to the conditions of the Creative Commons Attribution-NonCommercialNoDerivatives 4.0 International (CC BY-NC-ND 4.0) licence. Full details of this licence are available at: https://creativecommons.org/licenses/by-nc-nd/4.0/

\section{LICENCE}

CC BY-NC-ND 4.0

\section{REPOSITORY RECORD}

Lazauskas, Tomas, Steven D. Kenny, and Roger Smith. 2019. "Influence of the Prefactor to Defect Motion in Alpha-iron During Long Time Scale Simulations”. figshare. https://hdl.handle.net/2134/16928. 


\title{
Influence of the prefactor to defect motion in $\alpha$-Iron during long time scale simulations
}

\author{
T Lazauskas, SD Kenny and ${ }^{*} \mathbf{R}$ Smith \\ Department of Mathematical Sciences, Loughborough University, Leicestershire, \\ LE11 3TU, UK \\ E-mail: *r.smith@lboro.ac.uk
}

\begin{abstract}
We present a study of the influence of the prefactor in the Arrhenius equation for the long time scale motion of defects in $\alpha$-Fe. It is shown that calculated prefactors vary widely between different defect types and it is thus important to determine these accurately when implementing on-the-fly kinetic Monte Carlo (otfKMC) simulations. The results were verified by reproducing many events using Molecular Dynamics (MD) and Temperature-Accelerated Dynamics (TAD). The calculated prefactor was shown to increase the relative interstitial-vacancy diffusion rates by an order of magnitude compared to the assumption of a constant prefactor value and the ordering of the rate table for the interstitial defect migration mechanisms was also changed. In addition, low prefactor values were observed for the 4 interstitial dumbbells configuration with low barrier transitions.
\end{abstract}

Keywords: Arrhenius equation, prefactor, kinetic Monte Carlo, defect motion, bcc Fe

\section{Introduction}

Transition state theory (TST) operates on the idea of having transition rates between two states calculated considering only the equilibrium and saddle states of the transition by using the Arrhenius equation:

$$
r=\tau \exp \left(-\Delta E / k_{B} T\right),
$$

where $\Delta E$ is the activation energy, $k_{B}$ - Boltzmann's constant, $T$ - temperature and $\tau$ is the pre-exponential coefficient also known as the prefactor.

The Arrhenius equation is widely used in KMC simulations, but whereas techniques such as the nudged elastic band (NEB) or the Dimer method [1,2] have been used accurately to determine $\Delta E$, the calculation of the prefactor $\tau$ has come under less scrutiny. One of the first methods to carry out long time scale dynamics simulations within harmonic transition state theory (hTST) approximation was suggested by Henkelman and Jónsson [3]. They suggested to characterise systems by local energy minima, do multiple saddle searches and calculate rates for each individual transition using hTST and to select an event and advance the simulation clock according to the KMC algorithm. It has become widespread practise to use fixed $\tau$ values, usually ranging 
from $10^{12}$ to $10^{13} \mathrm{~s}^{-1}$, in such KMC simulations [4, 5, 6, 7], with the assumption that the attempt frequency does not vary greatly. This is because an accurate determination is computationally expensive and 'small' variations in the energy barrier $\Delta E$ affect the transition rates much more than 'small' variations in $\tau$. However recent work has found that an accurate calculation of the prefactor is necessary in order to include important defect diffusion mechanisms that would not be accessible if a constant value would be used. Examples of variations in prefactor values were also reported in other materials, such as, unusually high prefactors, up to $10^{19} \mathrm{~s}^{-1}$ for the surface changes on $\operatorname{InAs}(001)$ [8], the formation of a stacking fault tetrahedron in $\mathrm{Cu}[9]$ with the prefactor value of $10^{22} \mathrm{~s}^{-1}$ and the disassociation of $\mathrm{O}_{2}$ in $\mathrm{TiO}_{2}$ with the prefactor of $1.6 \times 10^{16} \mathrm{~s}^{-1}$ during the growth process [10].

In this paper we show that relative transition rates of the small defect clusters depend crucially on $\tau$, where $\tau$ for small defect clusters in $\alpha$-Fe, is determined using the Vineyard equation [11]:

$$
\tau=\frac{\prod_{j=1}^{N} v_{j}}{\prod_{j=1}^{N-1} v_{j}^{*}},
$$

here $v$ and $v^{*}$ are the normal frequencies for vibrations at the initial (local minimum) and saddle states respectively.

The Vineyard formula is only applicable at rank 1 saddle points. In all the cases considered in this paper, the saddle points were rank 1 . When searching for saddle points sometimes the chosen pathway is a rank 2 saddle. These must be treated separately either using the approach given in [12] or by determining the nearby rank 1 saddle by following the path of the second lowest eigenvalue of the Hessian which is the approach that we normally apply in our simulations [13].

The motivation for the study was an examination of the long term evolution of radiation damage in $\alpha$-Fe. It is well known that a collision cascade introduces point defects and small interstitial and vacancy clusters into a perfect lattice but here we isolate typical defects seen in collision cascade studies and examine their transitions in isolation. Thus defects, such as dumbbell interstitials (DB), di-interstitials, vancancies and di-vacancies were artificially introduced into the system to investigate their transition rate dependency on the prefactor value independently of their local environment. The defects' configurations and migration pathways are compared those determined in previous works by $[14,15,16]$.

\section{Methodology}

\subsection{System}

For our simulations we use an $\alpha$-Fe system described by the well known Ackland 2004 potential [17] containing up to 54,000 atoms with a system size of $30 a_{0} \times 30 a_{0} \times$ $30 a_{0}$, where $a_{0}$ is the lattice parameter. We calculate the diffusion rates between local 
minimum states at $450 \mathrm{~K}$. In some cases, the Mendelev 2003 [18], potential is also used for comparison, but if not stated the Ackland 2004 potential is used by default.

\subsection{Method}

To determine the various transition pathways a combination of the Dimer [2] and the minimum mode following [19] methods have been implemented into an otf-KMC technique [6] in order to achieve the best mixture of low computational costs and accuracy for finding the various saddle states surrounding a local minimum. The switch from the Dimer to the minimum mode method occurs when the Dimer's curvature value becomes negative and there is at least one negative eigenvalue in the system by which the minimum mode method can converge to a saddle. In some cases the calculated energy barriers were also determined by the NEB method when the final transition state was known.

As mentioned before, we use eq. 2 to calculate the prefactor for each transition we find during a KMC step. The normal frequencies used in the formula, at the initial and saddle states, are derived from the eigenvalues using e.q. 3. Eigenvalues are calculated by numerically constructing the Hessian $H$ (e.q. 4) and then applying the DSYEV routine from the Linear Algebra Package (LAPACK).

$$
v=\frac{\sqrt{\lambda}}{2 \pi},
$$

here, $\lambda$ is the eigenvalue from which the normal frequency $v$ is derived.

$$
H_{i, j}=\frac{1}{2 \sqrt{m_{i} m_{j}}}\left(\frac{F_{i}^{j+}-F_{i}^{j-}}{2 \delta}+\frac{F_{j}^{i+}-F_{j}^{i-}}{2 \delta}\right),
$$

where $F_{i}^{j+}$ is the force acting on $i$-th component due to the positive ('-' - negative) displacement in the $j$-th components position, $\delta$ is the displacement $(0.001 \AA)$ and $m_{i}$ is atomic mass of the $i$-th atom. The value of $\delta$ was chosen to be small enough so that the finite differencing approximates as accurately as possible the true derivative but big enough to avoid numerical instability due to computer rounding. Also, due to minor differences between the symmetric elements of $H$, the element $H_{i, j}$ is calculated as an average of $H_{i, j}$ and $H_{j, i}$.

\subsection{Performance}

In order to understand the relationship between the scaling of the computational time and the convergence of the prefactor value, a series of tests has been performed. To show the tendencies observed, a single vacancy defect system is given as an example. By changing the radius of the volume of atoms around the defect that are included into the calculation, we track the convergence of the prefactor value and the time taken to calculate the eigenvalues of the Hessian. The data is summarised in table 1.

In table 1 the first column represents the radius around the region of the vacancy defect, which determines how many atoms will be included in to the calculation; the 
Table 1: Calculated prefactor values as a function of the inclusion radius of the atoms around a vacancy defect, for the isolated vacancy diffusion process at $450 \mathrm{~K}$.

\begin{tabular}{|r|r|c|r|}
\hline $\begin{array}{r}\text { Radius } \\
(\AA)\end{array}$ & Atoms & $\begin{array}{c}\text { Prefactor } \\
\mathrm{s}^{-1}\end{array}$ & $\begin{array}{r}\text { Eigenvalues } \\
\text { calc. time }(\mathrm{s})\end{array}$ \\
\hline 2.0 & 14 & $1.42 \times 10^{13}$ & 6.48 \\
3.0 & 64 & $6.14 \times 10^{13}$ & 9.34 \\
5.0 & 160 & $8.10 \times 10^{13}$ & 37.85 \\
6.0 & 174 & $9.09 \times 10^{13}$ & 45.52 \\
7.0 & 306 & $1.00 \times 10^{14}$ & 112.05 \\
8.0 & 362 & $1.01 \times 10^{14}$ & 151.63 \\
10.0 & 640 & $1.04 \times 10^{14}$ & 404.86 \\
12.0 & 1042 & $1.06 \times 10^{14}$ & $1,004.60$ \\
14.0 & 1530 & $1.06 \times 10^{14}$ & $2,011.03$ \\
16.0 & 2204 & $1.07 \times 10^{14}$ & $4,063.14$ \\
18.0 & 3034 & $1.07 \times 10^{14}$ & $7,555.33$ \\
20.0 & 4020 & $1.07 \times 10^{14}$ & $13,309.30$ \\
\hline
\end{tabular}

prefactor values are calculated using eq. 2 and the time given is only for calculating eigenvalues at the initial state since it takes approximately the same amount of time to calculate eigenvalues at the initial state.

As can be seen from the results in table 1, to determine the prefactor to 3 figure accuracy requires over 2000 atoms, but in order to reach one, at least 306 atoms must be included and the calculation of one set of eigenvalues takes around 1/40 of the time. Therefore all the given data in the paper is calculated using a $7 \AA$ radius if it is not stated otherwise. This was regarded as the best compromise between accuracy and computational speed.

Even though it was reported by calculating point-defect entropy [20] that the embedded cluster method (which is equivalent to our approach of determining the size of the Hessian) is less accurate compared to the supercell method, the verification of the results, as we show in section 3.4, indicates that it works well in our cases.

\subsection{Vibrational contributions}

From the phonon density of states, vibrational contributions to the activation energy at finite temperature can be written as $[11,21]$

$$
\Delta F_{v i b}=\Delta U_{v i b}-T \Delta S_{v i b}
$$

where $U_{v i b}$ represents the vibrational internal energy and $S_{v i b}$ - the vibrational entropy.

When the prefactor is calculated using eq. 2, it incorporates only the vibrational entropy. In order to see whether the internal energy has an effect on the activation 
barrier, we used the expressions of these contributions as sum of single-oscillation contributions written as follows [21]:

$$
U_{v i b}=\sum_{i=1}^{3 N}\left[\frac{\hbar \lambda_{i}}{e^{\frac{\hbar \lambda}{k_{i} T}}-1}+\frac{1}{2} \hbar \lambda_{i}\right]
$$

and

$$
S_{v i b}=k_{B} \sum_{i=1}^{3 N}\left[\frac{\hbar \lambda_{i}}{k_{B} T}\left(e^{\frac{\hbar \lambda_{i}}{k_{B} T}}-1\right)^{-1}-\ln \left(1-e^{\frac{-\hbar \lambda_{i}}{k_{B} T}}\right)\right] .
$$

where $\hbar$ is the Planck's constant, $\lambda_{i}$ - the eigenvalue of $i$-th coordinate of the system and $T$ is the temperature.

We have calculated these contributions for the most common defects and their main migration transitions to check whether the vibrational internal energy has an effect on the activation barrier height. The summarised data is given in table 2. For these calculations we used a $23.0 \AA$ inclusion radius of atoms around the defect studied is taken to achieve good accuracy when calculating the normal frequencies. The vibrational internal energy's contribution does not change much, even when a smaller inclusion radius is used, such as $9.9 \AA$.

Table 2: Vibrational contributions to the barrier heights of the most common defects and their prime migration mechanisms at $450 \mathrm{~K}$. The last two entries represent diinterstitials with the same notation as introduced by Marinica et al. [16]. The DB transitions are defined in $\S 3.1$.

\begin{tabular}{|l|l|c|c|c|c|c|c|}
\hline \multirow{2}{*}{ Defect } & \multirow{2}{*}{ Migration } & \multicolumn{2}{|c|}{ Initial state $(\mathrm{eV})$} & \multicolumn{2}{|c|}{ Saddle state $(\mathrm{eV})$} & \multicolumn{2}{c|}{$\Delta(\mathrm{eV})$} \\
\cline { 3 - 8 } & & $U_{\text {vib }}$ & $T S_{\text {vib }}$ & $U_{\text {vib }}(\mathrm{eV})$ & $T S_{\text {vib }}$ & $U_{\text {vib }}$ & $T S_{v i b}$ \\
\hline$\langle 110\rangle$ DB & rot.-trans. & 481.6656 & 1574.0375 & 481.6655 & 1574.1324 & -0.0001 & 0.0949 \\
$\langle 110\rangle$ DB & on-site rot. & 481.6656 & 1574.0375 & 481.6653 & 1574.3079 & -0.0004 & 0.2704 \\
VAC & $1-\mathrm{NN}$ & 468.1563 & 1529.9442 & 486.1561 & 1530.0729 & -0.0002 & 0.1287 \\
$I_{2}^{110}$ & $I_{2}^{2 a}$ & 519.1650 & 1696.7286 & 519.1649 & 1696.8321 & -0.0001 & 0.1035 \\
$I_{2}^{110}$ & $I_{2}^{110}$ & 519.1650 & 1696.7286 & 519.1649 & 1696.8321 & -0.0001 & 0.1035 \\
\hline
\end{tabular}

As can be seen in table 2, the biggest contribution to the barrier height is due to the vibrational entropy which is included when the prefactor is calculated by using the Vineyard equation. The vibrational internal energy's contribution is negligible compared to the barrier heights for all the transitions investigated. Therefore, we conclude, that for the vibrational contributions, the Vineyard formula is accurate enough for our calculations.

\section{Results}

\subsection{Interstitials}

The case of interstitial defects and migration pathways are well studied, therefore we will only present the summarised data. In our simulations the $\langle 110\rangle \mathrm{DB}$ is the most common 
configuration occurring in collision cascade simulations, but the $\langle 111\rangle$ can appear for a short period during the collisional phase of a cascade. This is also confirmed by experimental findings [22] and the ab initio studies [15] on the stability and mobility of interstitials in $\alpha$-Fe, where studies predict a rapid change from the $\langle 111\rangle$ to $\langle 110\rangle \mathrm{DB}$. Therefore, we concentrate on studying the $\langle 110\rangle$ DB's migration rates rather than $\langle 111\rangle$. The data is summarised in table 3 . Here and in subsequent calculations the constant prefactor value is taken as $1 \times 10^{13} \mathrm{~s}^{-1}$.

Table 3: The $\langle 110\rangle$ DB migration rates at $450 \mathrm{~K}$.

\begin{tabular}{|l|r|c|r|r|}
\hline Mechanism & $\begin{array}{r}\text { Barrier } \\
(\mathrm{eV})\end{array}$ & $\begin{array}{c}\text { Prefactor } \\
\left(\mathrm{s}^{-1}\right)\end{array}$ & $\begin{array}{r}\text { Rate }\left(\mathrm{s}^{-1}\right) \\
\text { (const. prefactor) }\end{array}$ & $\begin{array}{r}\text { Rate }\left(\mathrm{s}^{-1}\right) \\
(\text { calc. prefactor) }\end{array}$ \\
\hline Translation-rotation & 0.31 & $1.8 \times 10^{13}$ & $3.0 \times 10^{9}$ & $5.5 \times 10^{9}$ \\
$\langle 110\rangle$ rotation & 0.43 & $7.5 \times 10^{14}$ & $1.5 \times 10^{8}$ & $1.1 \times 10^{10}$ \\
$\langle 110\rangle$ to octahedral & 0.69 & $3.4 \times 10^{13}$ & $1.7 \times 10^{5}$ & $5.9 \times 10^{5}$ \\
\hline
\end{tabular}

The first and the most common migration mechanism, is the one proposed by Johnson [14], a combination of translation and rotation. The migration barrier of 0.31 $\mathrm{eV}$ is in a very good agreement with the experimental value of $\approx 0.3 \mathrm{eV}$ by [22]. The calculated value of the prefactor $1.8 \times 10^{13} \mathrm{~s}^{-1}$ is close to the constant value, normally used in KMC simulations and therefore the rate calculated by using this constant prefactor is approximately the same order of magnitude as when calculated by using the Vineyard method.

A quite different situation can be seen for the $\langle 110\rangle$ DB on-site rotation between the $\langle 110\rangle$ directions represented by the second row in table 3 . Here rotation-transition has a barrier of $0.43 \mathrm{eV}$ with a prefactor value almost two orders of magnitude higher than the constant value.

The third case in the table represents the first nearest neighbour (NN) jump with a saddle point near the octahedral configuration with the migration barrier of $0.69 \mathrm{eV}$. It has very similar constant and calculated prefactor values as for the translation and rotation mechanism.

Both migration energy values for the $\langle 110\rangle$ DB on-site rotation and the 1st NN jump through the tetrahedral configuration are almost equal to those that were calculated by Marinica et al. [16] by using the ARTn method to explore the energy landscape.

The two migration pathways from the most stable $\langle 110\rangle \mathrm{DB}$ configuration are the main mechanisms for mono interstitial migration. Also, implementation of a slightly different potential energy function [18] in our KMC technique did not have any significant change in barrier heights, rate values or set of possible transitions for the $\langle 110\rangle$ DB.

By using the constant prefactor value, the migration of the $\langle 110\rangle \mathrm{DB}$ is dominated by the translation-rotation mechanism, where the calculated prefactor value moves the on-site rotation transition to the top in the rate table and is thus more likely to be chosen in a KMC simulation. 


\subsection{Di-interstitials}

The lowest-energy configurations of di-interstitials reported by Marinica et al. [16] are studied in order to have a better understanding of migration mechanisms of these common defects in ballistically affected systems. The Mendelev 2003 and Ackland 2004 potentials give almost the same formation energies where the configuration of two nearest neighbour parallel $\langle 110\rangle$ DBs has the lowest energy. Later, we will use the same notation for the lowest di-interstitial configurations as introduced by Marinica et al. The data for the migration mechanisms of one of the most common di-interstitial configuration $I_{2}^{<110>}$ is summarised in table 4 .

Table 4: Migration rates of the $I_{2}^{<110>}$ di-interstitial at $450 \mathrm{~K}$.

\begin{tabular}{|l|r|c|r|r|}
\hline Config. & $\begin{array}{r}\text { Barrier } \\
(\mathrm{eV})\end{array}$ & $\begin{array}{r}\text { Prefactor } \\
\left(\mathrm{s}^{-1}\right)\end{array}$ & $\begin{array}{r}\text { Rate }\left(\mathrm{s}^{-1}\right) \\
\text { (const. prefactor) }\end{array}$ & $\begin{array}{r}\text { Rate }\left(\mathrm{s}^{-1}\right) \\
\text { (calc. prefactor) }\end{array}$ \\
\hline$I_{2}^{2 a}$ & 0.33 & $1.2 \times 10^{13}$ & $2.0 \times 10^{9}$ & $2.4 \times 10^{9}$ \\
$I_{2}^{<110>}$ & 0.36 & $1.4 \times 10^{13}$ & $9.3 \times 10^{8}$ & $1.3 \times 10^{9}$ \\
$I_{2}^{<110>}$ & 0.49 & $9.4 \times 10^{12}$ & $3.3 \times 10^{7}$ & $3.1 \times 10^{7}$ \\
$I_{2}^{5}$ & 0.48 & $5.8 \times 10^{13}$ & $4.2 \times 10^{7}$ & $2.4 \times 10^{8}$ \\
$I_{2}^{6 a}$ & 0.50 & $3.0 \times 10^{13}$ & $2.5 \times 10^{7}$ & $7.2 \times 10^{7}$ \\
\hline
\end{tabular}

In table 4 we present only those migration mechanisms from the $I_{2}^{<110>}$ configuration that have highest rate (constant and calculated) values, since for di-interstitials there is usually more than one migration pathway between configurations. In all the cases we can see that calculated prefactor value is close to the constant value and has a minor effect on the rate table. Only migration to the $I_{2}^{5}$ configuration was found to have a prefactor that differed appreciably, at $5.8 \times 10^{13}$ which is five times greater than the constant one and brings this migration closer to the the migrations to $I_{2}^{2 a}$ and $I_{2}^{<110>}$ with barrier heights of $0.33 \mathrm{eV}$ and $0.36 \mathrm{eV}$ in terms of the rate values.

We also examined the most stable di-interstitial configurations that were found by our KMC technique from other configurations, such as $I_{2}^{<110>}, I_{2}^{5}, I_{2}^{6 a}$. As in the previous example the calculated prefactor does not vary greatly and in most of the cases fluctuates between $0.5-5.0 \times 10^{13} \mathrm{~s}^{-1}$. One of the more interesting cases was seen for $I_{2}^{2 a}$ diffusion to $I_{2}^{<110>}$ by two possible pathways with barriers of $0.09 \mathrm{eV}$ and 0.25 $\mathrm{eV}$. Both pathways have very similar rate values $\left(1.1 \times 10^{12} \mathrm{~s}^{-1}, 1.3 \times 10^{12} \mathrm{~s}^{-1}\right)$ due to the estimated prefactors of $1.1 \times 10^{13} \mathrm{~s}^{-1}$ and $8.0 \times 10^{14} \mathrm{~s}^{-1}$, thus making the diffusion even more favourable.

To sum up the behaviour of the di-interstitial migration, it is clear that the $I_{2}^{<110>}$ configuration dominates with an occasional jump to other configurations, but it is not likely for the atoms to stay in these configurations for a long time and by couple of intermediate configurations can return to $I_{2}^{<110>}$. 


\subsection{Vacancies}

The second most common defect after a radiation event is a vacancy. Its migration energy is $0.64 \mathrm{eV}$ and has a minor dip at the midpoint, therefore the migration mechanism has two saddle points as was shown by Johnson [14]. The configuration at the midstate is metastable due to very low energy difference of $0.04 \mathrm{eV}$. Vacancy migration gives a prefactor of $1.0 \times 10^{14} \mathrm{~s}^{-1}$, an order of magnitude greater than the constant value, as is shown in table 5 . The higher rate value with the calculated prefactor makes the vacancy - interstitial diffusion ratio change by an order of magnitude compared to the assumption of a fixed prefactor, thus making the vacancy migration more attainable by the KMC algorithm when there are other type of defects with lower barriers and/or higher rate migrations in the simulation box.

Table 5: The migration rates of a vacancy defect at $450 \mathrm{~K}$.

\begin{tabular}{|l|r|c|r|r|}
\hline Mig. type & $\begin{array}{r}\text { Barrier } \\
(\mathrm{eV})\end{array}$ & $\begin{array}{r}\text { Prefactor } \\
\left(\mathrm{s}^{-1}\right)\end{array}$ & $\begin{array}{r}\text { Rate }\left(\mathrm{s}^{-1}\right) \\
\text { (const. prefactor) }\end{array}$ & $\begin{array}{r}\text { Rate }\left(\mathrm{s}^{-1}\right) \\
\text { (calc. prefactor) }\end{array}$ \\
\hline 1-NN jump & 0.64 & $1.0 \times 10^{14}$ & $6.42 \times 10^{5}$ & $6.43 \times 10^{6}$ \\
2-NN jump & 2.61 & $1.8 \times 10^{14}$ & $5.8 \times 10^{-17}$ & $1.0 \times 10^{-15}$ \\
\hline
\end{tabular}

The di-vacancy migration process is a step-wise process whereby one of the two vacancies first moves, by jumping to one of its 1st NN neighbours. The migration has a slight depression at the midpoint as in the previous example. All the observed processes for the di-vacancy defect greatly depend on the initial configuration of two vacancies.

In this work we have studied four different di-vacancy configurations where two vacancies are situated by separating them from the first to the fourth NN positions.

As can be seen in table 6, the calculated prefactor does not change the ordering of the rate table and the migration transitions with the lowest barriers, whether the prefactor is calculated or the constant value is used, have the highest rate. However in all the cases the calculated prefactor is higher than the constant value and in most of the cases by at least one order of magnitude. Table 6 also shows that di-vacancy migration is mainly carried out through 1, 2, $4 \mathrm{NN}$ configurations due to the highest rate values.

\subsection{Verifying rate values}

In order to check the estimated rates with the calculated prefactor, we have carried out a series of tests. We have reproduced the same migration mechanisms we saw in KMC simulations by evolving the systems with MD as follows: we begin with a system that has only one defect at its centre, whose migration rates are being studied. Then the system is thermalised up $450 \mathrm{~K}$ (the same temperature as used in the KMC) by applying the Berendsen thermostat [23] for 10 ps of simulation time. After that we evolve the system with regular MD until it crosses a saddle point separating two local 
Table 6: The calculated rates of the most common migration mechanisms of di-vacancy defects at $450 \mathrm{~K}$.

\begin{tabular}{|l|l|r|r|r|r|}
\hline $\begin{array}{l}\text { Initial } \\
\text { config. }\end{array}$ & $\begin{array}{l}\text { Final } \\
\text { config. }\end{array}$ & $\begin{array}{r}\text { Barrier } \\
(\mathrm{eV})\end{array}$ & $\begin{array}{r}\text { Prefactor } \\
\left(\mathrm{s}^{-1}\right)\end{array}$ & $\begin{array}{r}\text { Rate }\left(\mathrm{s}^{-1}\right) \\
(\text { const. prefactor })\end{array}$ & $\begin{array}{r}\left.\text { Rate } \mathrm{s}^{-1}\right) \\
\text { (calc. prefactor) }\end{array}$ \\
\hline $1 \mathrm{NN}$ & $2 \mathrm{NN}$ & 0.62 & $1.5 \times 10^{14}$ & $1.1 \times 10^{6}$ & $1.7 \times 10^{7}$ \\
$1 \mathrm{NN}$ & $3 \mathrm{NN}$ & 0.71 & $3.9 \times 10^{13}$ & $1.1 \times 10^{5}$ & $4.4 \times 10^{5}$ \\
$1 \mathrm{NN}$ & $5 \mathrm{NN}$ & 0.66 & $1.0 \times 10^{14}$ & $4.1 \times 10^{5}$ & $4.1 \times 10^{6}$ \\
\hline $2 \mathrm{NN}$ & $1 \mathrm{NN}$ & 0.57 & $3.1 \times 10^{13}$ & $1.1 \times 10^{6}$ & $1.3 \times 10^{7}$ \\
$2 \mathrm{NN}$ & $4 \mathrm{NN}$ & 0.63 & $1.2 \times 10^{14}$ & $8.8 \times 10^{5}$ & $1.1 \times 10^{7}$ \\
\hline $3 \mathrm{NN}$ & $1 \mathrm{NN}$ & 0.67 & $6.1 \times 10^{13}$ & $3.1 \times 10^{5}$ & $1.9 \times 10^{6}$ \\
$3 \mathrm{NN}$ & $4 \mathrm{NN}$ & 0.63 & $1.3 \times 10^{14}$ & $8.8 \times 10^{5}$ & $1.1 \times 10^{7}$ \\
$3 \mathrm{NN}$ & $7 \mathrm{NN}$ & 0.66 & $1.0 \times 10^{14}$ & $4.1 \times 10^{5}$ & $4.1 \times 10^{6}$ \\
\hline $4 \mathrm{NN}$ & $2 \mathrm{NN}$ & 0.43 & $1.0 \times 10^{14}$ & $1.5 \times 10^{8}$ & $1.5 \times 10^{9}$ \\
$4 \mathrm{NN}$ & $3 \mathrm{NN}$ & 0.64 & $8.7 \times 10^{13}$ & $6.8 \times 10^{5}$ & $5.9 \times 10^{6}$ \\
$4 \mathrm{NN}$ & $5 \mathrm{NN}$ & 0.59 & $1.2 \times 10^{14}$ & $2.5 \times 10^{6}$ & $3.0 \times 10^{7}$ \\
$4 \mathrm{NN}$ & $6 \mathrm{NN}$ & 0.67 & $7.3 \times 10^{13}$ & $3.1 \times 10^{5}$ & $2.3 \times 10^{6}$ \\
$4 \mathrm{NN}$ & $8 \mathrm{NN}$ & 0.61 & $1.2 \times 10^{14}$ & $1.5 \times 10^{6}$ & $1.8 \times 10^{7}$ \\
$4 \mathrm{NN}$ & $9 \mathrm{NN}$ & 0.64 & $1.0 \times 10^{14}$ & $6.8 \times 10^{5}$ & $6.8 \times 10^{6}$ \\
\hline
\end{tabular}

energy minima. We capture this moment by checking the dot product of the force vector of the system and the atom separation vector between the current and the initial (just after thermalisation) states. If the dot product is positive it means that both vectors are pointing to the same direction and we have crossed the saddle. The time taken for the system to migrate is measured and the new minimum state is found by relaxing the system by the Conjugate Gradient method. We have carried out a set of 1,000 such transitions for the DB interstitial and the single vacancy, 10,000 test simulations for di-interstitial defects and analysed the results according to migration mechanisms seen in the KMC simulations.

For the vacancy defect we had to increase the temperature to $750 \mathrm{~K}$ in order to find transitions using the MD technique due to the time scales of the migration mechanisms and then recalculate simulation times at $450 \mathrm{~K}$ by using eq. 8 as in the TAD method [24]:

$$
t_{\text {low }}=t_{\text {high }} \exp \left(\Delta E\left(\beta_{\text {low }}-\beta_{\text {high }}\right)\right) \text {, }
$$

where $t_{\text {high }}$ is actual simulation times at the higher $(750 \mathrm{~K})$ temperature and $t_{\text {low }}$ is the estimated simulation time at the lower $(450 \mathrm{~K})$ temperature, $\Delta E$ is the migration energy barrier, and $\beta$ is equal to $1 /\left(k_{B} T\right)$.

The results are compared with KMC results in terms of the prefactor value. The prefactor value is calculated as follows: first we calculate the rate $R$ of the specific migration mechanism by inverting the estimated the average simulation time $t_{\text {avg }}$ for it 
to happen multiplied by a number possible pathways $N_{\text {paths }}$ and converting the results into seconds:

$$
r=\frac{1}{t_{\text {avg }} * N_{\text {paths }}} * 10^{15}
$$

Then, using this rate value in eq. 1 with an appropriate $\Delta E$ value, calculated by using the climbing image NEB technique [1]), we estimate the prefactor value $\tau$. The results for the interstitial, di-interstitial and vacancy defects are given in table 7 .

Table 7: Comparison of the rates and prefactor values for the common defects and their main migration pathways between $\mathrm{MD}$ and $\mathrm{KMC}$ simulations at $450 \mathrm{~K}$. In column $\%$ we show the percentage occurrence of the particular mechanism with respect to the total number of simulations and in the column \%rsd we measure the percentage of the relative standard error of the estimated value.

\begin{tabular}{|l|r|r|r|r|r|r|r|}
\hline \multirow{2}{*}{ Migration } & Barrier & \multicolumn{9}{|c|}{ MD } & \multicolumn{2}{|c|}{ KMC } \\
\cline { 3 - 8 } & $(\mathrm{eV})$ & $\%$ & $\tau\left(\mathrm{s}^{-1}\right)$ & $\mathrm{r}\left(\mathrm{s}^{-1}\right)$ & $\% \mathrm{rsd}$ & $\tau\left(\mathrm{s}^{-1}\right)$ & $\mathrm{r}\left(\mathrm{s}^{-1}\right)$ \\
\hline \multicolumn{8}{|c|}{$<110>\mathrm{DB}$} \\
\hline rot.-trans. & 0.31 & $56 \%$ & $3.0 \times 10^{13}$ & $1.1 \times 10^{10}$ & 4.1 & $1.8 \times 10^{13}$ & $6.5 \times 10^{9}$ \\
reorient. & 0.43 & $12 \%$ & $1.0 \times 10^{15}$ & $1.8 \times 10^{10}$ & 8.2 & $7.5 \times 10^{14}$ & $1.3 \times 10^{10}$ \\
\hline \multicolumn{8}{|c|}{$I_{2}^{<110>}$} \\
\hline$I_{2}^{2 a}$ & 0.32 & $47 \%$ & $2.6 \times 10^{13}$ & $7.2 \times 10^{9}$ & 1.3 & $1.2 \times 10^{13}$ & $3.2 \times 10^{9}$ \\
$I_{2}^{<110>}$ & 0.32 & $16 \%$ & $3.5 \times 10^{13}$ & $9.8 \times 10^{9}$ & 2.9 & $1.4 \times 10^{13}$ & $3.9 \times 10^{9}$ \\
\hline \multicolumn{8}{|c}{ VAC } \\
\hline 1-NN & 0.62 & $66 \%$ & $2.9 \times 10^{13}$ & $3.4 \times 10^{6}$ & 6.3 & $1.0 \times 10^{14}$ & $1.2 \times 10^{7}$ \\
\hline
\end{tabular}

Results show that there is a reasonable agreement between the MD and KMC techniques and the estimated prefactor from using Vineyard method is a good approximation for the one that was determined from the MD simulations. For the $<110>\mathrm{DB}$ and $I_{2}^{<110>}$ defects we see a difference in the prefactor values for all the migration mechanisms. This may occur because it is sometimes possible for the system to return to the same local energy minimum, even if the saddle state was crossed, where in our simulations it is not allowed. In this way the average simulation time maybe slightly shorter, thus giving a higher prefactor value for the migration mechanism. The same conclusion can be applied to the vacancy 1-NN jump migration only partially. The difference between the prefactor values is also caused by the higher $(750 \mathrm{~K})$ temperature at which simulations were carried out where the harmonic approximation breaks down. To estimate the level of the error we additionally carried out simulations for the [110] $D B$ at 550 and $650 \mathrm{~K}$ temperatures (see table 8) and checked the prefactor values for both most common migrations recalculated at $450 \mathrm{~K}$. We can clearly see that for higher temperature the prefactor value at lower temperature is underestimated, thus the MD results for the vacancy defect are also likely to be underestimated. 
Table 8: [110] The DB migration prefactor values calculated at $T_{\text {low }}=450 \mathrm{~K}$.

\begin{tabular}{|r|r|r|}
\hline \multirow{2}{*}{$T_{\text {high }}(\mathrm{K})$} & \multicolumn{2}{|l|}{ Prefactor value $\left(\mathrm{s}^{-1}\right)$ at $T_{\text {low }}$ for } \\
\cline { 2 - 3 } & rot.-trans. & reorrient. \\
\hline 450 & $3.0 \times 10^{13}$ & $1.0 \times 10^{15}$ \\
550 & $2.5 \times 10^{13}$ & $5.1 \times 10^{14}$ \\
650 & $1.8 \times 10^{13}$ & $2.3 \times 10^{14}$ \\
\hline
\end{tabular}

\subsection{Influence of the prefactor during long-time scale simulations of collision cascade evolution}

In order to investigate, how an accurate determination of the prefactor may influence otf-KMC simulations, we have carried out simulations on defect configurations that were initially produced by a radiation event. $66,1 \mathrm{keV}$ cascades were carried out in the same way as described in [25]. Of these, 10 representative cases were chosen and further evolved by otf-KMC using the constant and calculated prefactors. The cases were chosen in such a manner, that they would represent different defect configurations and distributions in the system in order to provide a good insight of the processes.

Usually, the outcome of a collision cascade simulation consists of a vacancy rich region close to the initial PKA site surrounded by outlying interstitials. Therefore, we start by comparing the behaviour of the defects and then the effects on the whole system in terms of energetics and defect numbers.

Due to the low barrier of the migration processes of the $\langle 110\rangle \mathrm{DB}$, it dominates the initial phase of the simulations, but the migration mechanism is different between the constant and calculated cases. Simulations with the constant prefactor are driven by the rotation-translation of the $\langle 110\rangle \mathrm{DB}$, where the calculated case switches between reorientation, which has a higher rate value and the rotation-translation mechanisms. Therefore, in the simulation with the constant prefactor, $\langle 110\rangle$ DBs tend to explore the system more, whereas in the other case the $\langle 110\rangle$ is not so mobile. In the first case, it is more likely that $\langle 110\rangle \mathrm{DB}$ will find another defect to recombine or to cluster faster by the exploring the system, whereas in the second case, if there are other defects in the vicinity of the $\langle 110\rangle \mathrm{DB}$, through reorientation, the DB is more likely to position itself in a direction towards the defects before migration towards them.

A clear effect of the accurately determined prefactor can also be seen for the vacancy type defects. With the constant prefactor used in the simulations, vacancy defect migrations are observed quite rarely, only several in couple of thousand KMC steps, if mobile interstitials are present in the system as well. With the calculated prefactor, we can see vacancy defect jumps more often, due to the higher rate values. Thus, we are able to see that vacancies tend to cluster and rearrange in to highly symmetrical (Fig. 1) configurations compared to using a constant value. On average the simulation time for a vacancy type defect to migrate with calculated prefactor is $\approx 3 \times 10^{-9} s$, where the time with a constant value is almost an order of magnitude, longer $\approx 4 \times 10^{-8} s$. 
These values were calculated from the evolution of 8 systems, evolved for more than 1,000 KMC steps with calculated and constant prefactors.

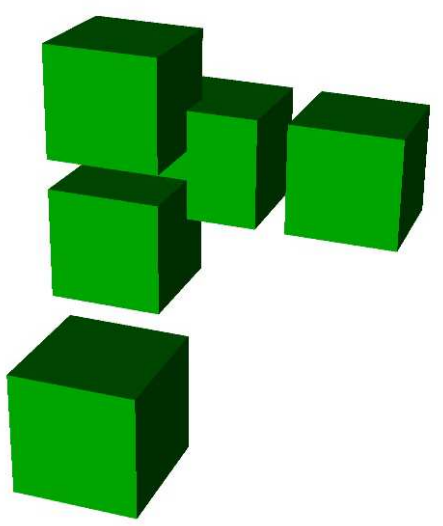

(a) Initial

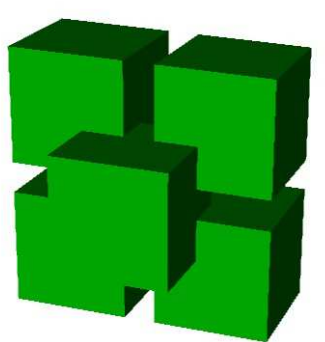

(b) Cluster

Figure 1: Clustering of 5 vacancies. a) Initial distribution of vacancies, post-cascade configuration, b) High symmetry 5 vacancy cluster after 75 KMC steps

By counting the number of chosen transitions with a prefactor value that is $\leq 10^{12}$ $\mathrm{s}^{-1}$ or $\geq 10^{14} \mathrm{~s}^{-1}$ against the total number of KMC steps within a simulation we can check if the processes that drive the KMC simulation depend on the calculation of the prefactor. We checked nine simulations that were carried out for at least 1,000 KMC steps and on average over $50 \%$ of the migration jumps had a prefactor value which is $\leq 10^{12} \mathrm{~s}^{-1}$ or $\geq 10^{14} \mathrm{~s}^{-1}$. In the cases where systems contain widely separated $\langle 110\rangle$ DBs and vacancies, the percentage of chosen migrations affected by the calculation of prefactors is quite high $\approx 70 \%$, where in the cases with di-, tri-interstitials, it is the opposite with the percentage $\approx 30 \%$.

It was observed that accurate prefactor calculations also slightly influence the recombination of defects. If an interstitial type defect lies a few $\mathrm{NN}$ away from a vacancy type defect, these two are more likely to recombine with the calculated prefactor. To investigate this situation further, a vacancy and $\langle 110\rangle$ DB defects were initially created in defect-free system by changing the separation between them and evolving these system by using otf-KMC. The cases of $1 \mathrm{NN}$ and $2 \mathrm{NN}$ separation are quite straightforward where recombination transitions have the lowest barriers and highest rates. The $3 \mathrm{NN}$ separation case is where the calculated prefactor does have an effect on the rate table. The lowest barrier $(\sim 0.29 \mathrm{eV})$ transitions are jumps to $4 \mathrm{NN}$ separation configurations with a $1.9 \times 10^{10} \mathrm{~s}^{-1}$ rate value, whereas a recombination transition with a slightly higher barrier of $0.36 \mathrm{eV}$ has an order of magnitude higher rate value $2.7 \times 10^{11} \mathrm{~s}^{-1}$ with a prefactor of $3.1 \times 10^{15} \mathrm{~s}^{-1}$, thus making this recombination more favourable. When a vacancy and a $\langle 110\rangle \mathrm{DB}$ defect are initially separated by the $4 \mathrm{NN}$ distance, recombination occurs as quickly as in the $1 \mathrm{NN}$ and $2 \mathrm{NN}$ cases, due to a low barrier $(0.22$ $\mathrm{eV}$ ) transition. The $5 \mathrm{NN}$ case is vary similar to the $3 \mathrm{NN}$ case; the lowest transitions 
with a barrier of $0.25 \mathrm{eV}$ and a rate value of $2.2 \times 10^{10} \mathrm{~s}^{-1}$, are jumps to the $4 \mathrm{NN}$ configuration and a slightly higher barrier $(0.33 \mathrm{eV})$ recombination transition has an order of magnitude higher rate value of $1.5 \times 10^{11} \mathrm{~s}^{-1}$ and is thus preferred. In the bigger separation cases, $6 \mathrm{NN}$ and more, the prefactor influence was not sufficient to influence the recombination processes.

Calculation of the prefactor has an influence on the bigger defect clusters too. One of the cases observed is the four interstitial cluster (Fig. 2 (a)). In this case a few different transitions were found for this cluster to re-configure with barriers ranging between $0.1-0.2 \mathrm{eV}$. The calculated prefactors are at least one order of magnitude lower than a constant value, e.g. to reconfigure from 2 (a) to 2 (b), a barrier of $0.1 \mathrm{eV}$ must be crossed with $1.8 \times 10^{11} \mathrm{~s}^{-1}$ prefactor which leads to a rate value of $1.2 \times 10^{10} \mathrm{~s}^{-1}$. The calculated prefactor puts this transition on the same time scale with smaller interstitial defects, and will not dominate the rate table, as it would, if the constant value was to be used.

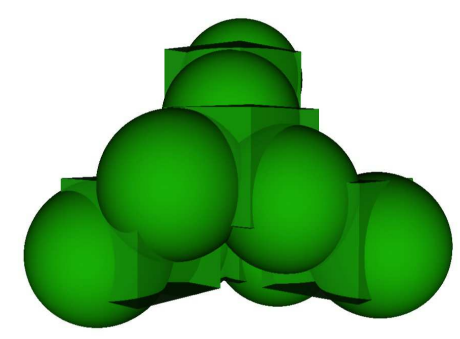

(a) Initial

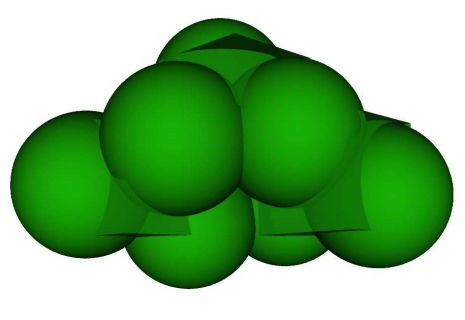

(b) Reconfigured

Figure 2: The interstitial cluster formed of 4 DBs. a) Initial, post-cascade configuration, b) The reconfigured structure after crossing a $0.1 \mathrm{eV}$ barrier.

Another configuration of the four interstitial cluster demonstrates an example of the prefactor influencing defect recombination. In this case four split interstitials are located in 1st NN positions and aligned in a slightly tilted $\langle 111\rangle$ configuration (Fig. 3 (a)). In this case, the defect cluster's centre of mass (com) is approximately $15 \AA$ away from a four vacancy cluster's com (Fig. 3 (b)). The migration transitions for this interstitial cluster along the $\langle 111\rangle$ direction have barrier heights ranging from 0.20 to $0.30 \mathrm{eV}$ with the prefactor values varying from $1.0 \times 10^{14} \mathrm{~s}^{-1}$ to $5.0 \times 10^{18} \mathrm{~s}^{-1}$. The higher prefactor values are estimated when migration is happening towards the vacancies cluster, making this migration process more accessible by the KMC technique and after a couple hundreds of KMC steps these two clusters recombine.

\section{Conclusions}

We have applied the Vineyard equation to determine the influence of an accurately calculated prefactor on the diffusion of small defect clusters in $\alpha$-Fe. The contribution of the vibrational internal energy, which is not incorporated within the Vineyard equation, 


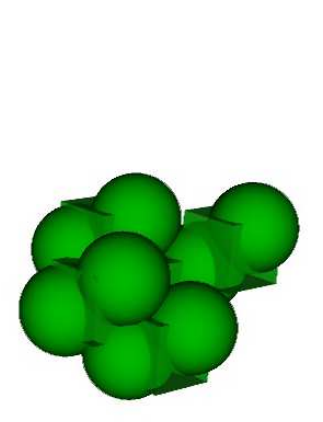

(a) 4 DB cluster
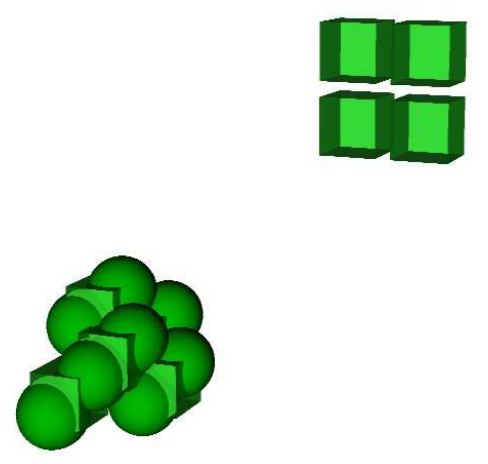

(b) DBs and Vacs clusters

Figure 3: A highly mobile split interstitial cluster, (a) containing four slightly tilted $\langle 111\rangle$ DBs in 1st NN positions, (b) the DBs cluster in the vicinity of four vacancies cluster.

showed only a minute contribution to the barrier height for the main defects types and their key migration mechanisms. In order to achieve one significant figure accuracy for the prefactor, it is necessary to include atoms at least within $7 \AA$ radius around the defect, which results in computations that are not excessive in terms of computing time. Results for the $\langle 110\rangle$ DB showed an almost two orders of magnitude greater prefactor value for the $\langle 111\rangle \mathrm{DB}$ on-site rotation, compared to a constant value, making it the fastest transition and more likely to be chosen than the translation-rotation mechanism that has a lower barrier height. For most of the transitions of the vacancy type defects, single and di-vacancy, the prefactor value is a least an order magnitude greater than the constant value, thus reducing the difference between the diffusion rates of interstitial and vacancy type defects. The results show that an accurate determination of the prefactor, in addition to the transition energy barriers, is necessary for accurate prediction of defect motion in KMC simulations.

\section{Acknowledgements}

The work was funded by EPSRC Grant EP/I012400/1 with Chris Grovenor of Oxford University as lead investigator. Thanks go to him and Dr. C.S. Sundar of IGCAR, India for useful discussions.

\section{References}

[1] Henkelman G, Uberuaga B P and Jónsson H 2000 The Journal of Chemical Physics 113 9901-9904

[2] Henkelman G and Jónsson H 1999 The Journal of Chemical Physics 111 7010-7022

[3] Henkelman G and Jónsson H 2001 The Journal of Chemical Physics 1159657 ISSN 00219606

[4] Voter A F 2007 Introduction to the kinetic Monte Carlo method Radiation Effects in Solids vol 235 (Springer Netherlands) chap 1, pp 1-23 ISBN 978-1-4020-5293-4

[5] El-Mellouhi F, Mousseau N and Lewis L J 2008 Physical Review B 78 1-4 ISSN 1098-0121

[6] Scott C, Blackwell S, Vernon L, Kenny S, Walls M and Smith R 2011 The Journal of Chemical Physics 135174706 ISSN 1089-7690 
[7] Xu H, Stoller R E and Osetsky Y N 2013 Journal of Nuclear Materials 443 66-70 ISSN 00223115

[8] Grosse F, Barvosa-Carter W, Zinck J J and Gyure M F 2002 Physical Review B 66075321.

[9] Uberuaga B P, Hoagland R G, Voter A F and Valone S M 2007 Physical Review Letters 99135501 ISSN 0031-9007

[10] Vernon L J, PhD Thesis, Loughborough University, 2010

[11] Vineyard G H 1957 Journal of Physics and Chemistry of Solids 3 121-127

[12] Kolluri K, Demkowicz M J 2012 Physical Review B 85205416

[13] Kittiratanawasin L, Smith R 2011 Nuclear Instruments and Methods in Physics Research B 269 17121719

[14] Johnson R A 1964 Physical Review 134 A1329-A1336

[15] Fu C C, Willaime F and Ordejón P 2004 Physical Review Letters 92175503 ISSN 0031-9007

[16] Marinica M C, Willaime F and Mousseau N 2011 Physical Review B 83094119 ISSN 1098-0121

[17] Ackland G J, Mendelev M I, Srolovitz D J, Han S and Barashev A V 2004 Journal of Physics: Condensed Matter 16 S2629-S2642 ISSN 0953-8984

[18] Mendelev M I, Han S, Srolovitz D J, Ackland G J, Sun D Y and Asta M 2003 Philosophical Magazine 83 3977-3994 ISSN 1478-6435

[19] Pedersen A, Hafstein S F and Jónsson H 2011 SIAM Journal on Scientific Computing 33 633-652 ISSN 10648275

[20] Mishin Y, Sørensen M R and Voter A F 2001 Philosophical Magazine A 8125912612

[21] Lucas G and Schäublin R 2009 Nuclear Instruments and Methods in Physics Research B 267 3009-3012 ISSN 0168583X

[22] Schultz H 1991 Materials Science and Engineering: A 141 149-167

[23] Berendsen H J C, Postma J P M, van Gunsteren W F, DiNola A and Haak J R 1984 The Journal of Chemical Physics 813684 ISSN 00219606

[24] Sørensen M R and Voter A F 2000 The Journal of Chemical Physics 112 9599-9606 ISSN 00219606

[25] Lazauskas T, Kenny S D, Smith R, Nagra G, Dholakia M and Valsakumar M C 2013 Journal of Nuclear Materials 437 317-325 ISSN 00223115 